\title{
Transformations des filières françaises de produits carnés et laitiers : la place des éleveurs en question
}

INRA Prod. Anim., 2018, $31(1), 69-82$

Marie-Odile NOZIĖRES-PETIT' , Virginie BARITAUX², Christèle. COUZY ${ }^{3}$, Marie DERVILLÉ ${ }^{4}$, Christophe PERROT ${ }^{5}$, Pierre SANS 6 , Gérard YOU ${ }^{5}$

${ }^{1}$ Inra, UMR Selmet, 2 place Viala, 34060, Montpellier, France

2 Université Clermont Auvergne, AgroParisTech, Inra, Irstea, VetAgro Sup, UMR Territoires, 63170, Aubière, France

${ }^{3}$ Institut de l'Élevage, Agrapole, 23 rue Baldassini, 69364, Lyon, France

${ }^{4}$ LEREPS, Université de Toulouse, ENSFEA, IEP, 31042, Toulouse, France

${ }^{5}$ Institut de l'Élevage, 149 rue de Bercy, 75012, Paris, France

${ }^{6}$ ALISS UR1303, Université de Toulouse, Inra, ENVT, 31076, Toulouse, France

Couriel : marie-odile.nozieres-petit@inra.fr

Les opérateurs des filières de produits carnés et laitiers se recomposent fortement, en lien avec les transformations des modes de consommation et les évolutions des politiques publiques. Ces transformations réinterrogent les possibilités d'action des éleveurs pour participer à la structuration des filières et à la segmentation de l'offre auprès du consommateur.

\section{Introduction}

Les nouvelles attentes sociétales et les controverses concernant les secteurs de productions de ruminants (Delanoue et Roguet, 2015) ont modifié profondément les comportements de consommation et la demande de produits carnés et laitiers (Laisney, 2011). Ces cinquante dernières années ont été aussi marquées par la mise en place, puis les évolutions de la Politique Agricole Commune (PAC) et des politiques structurant les marchés, français et européens et par les transformations contrastées des filières françaises viandes bovine et ovine, laits de vache, de brebis et de chèvre. Cet article de synthèse a pour objectif d'analyser ces changements, à l'œuvre en France, en adoptant une perspective historique depuis les années 1960-1970, ainsi que de comprendre quelle y est la place des éleveurs. Pour cela, nous nous appuyons sur un ensemble de ressources bibliographiques (académiques et professionnelles) et sur des cas d'études conduites sur les transformations des filières. Nous analyserons, dans les deux premières parties, quelles sont les évolutions en matière de consommation et de politiques publiques structurant les marchés. Dans la troisième partie, nous verrons comment ces évolutions ont conduit à des modifications profondes dans les stratégies des acteurs des filières (concentration, évolution des modes de coordination...), à tous les niveaux (collecte, transformation, distribution). Dans les quatrième et cinquième parties, nous examinerons quelle est la place des éleveurs au sein de ces recompositions, en particulier dans la gouvernance et la structuration des filières, d'une part, et dans la segmentation de l'offre au consommateur, d'autre part.

\section{Une demande de produits laitiers et carnés qui se transforme}

Entre 1970 et la fin des années 1990, la consommation des produits carnés, comme celle des produits laitiers, progresse à un rythme supérieur à celui de la population française, ce qui se traduit par un accroissement très sensible de la consommation par habitant. Ainsi la consommation française, toutes viandes confondues, augmente de 77,6 en 1970 à 94,1 kg équivalent carcasse par habitant et par an (kgec/hab/an) en 1998 (tableau 1). La même évolution s'observe pour les produits laitiers dans leur ensemble (Nichèle et al., 2005). En revanche, entre 1998 et 2015, la consommation totale de produits carnés par habitant s'est affaissée de près de $6 \mathrm{kgec} / \mathrm{hab} / \mathrm{an}$, suivie, 
à partir du courant des années 2000, par celle des produits laitiers (tableau 1 et 2). Néanmoins, pour les produits carnés, cet affaissement de la consommation n'altère pas le fait que, depuis les années 1960, ils restent le premier poste de dépense d'alimentation des Français, constituant environ $25 \%$ des dépenses alimentaires hors boissons en 2016 (données Insee in Agreste, 2017).

Au sein de ces deux grandes catégories de produits (laitiers et carnés), les évolutions des consommations diffèrent significativement. Certes, pour chacun des produits carnés (à l'exception du cheval), les volumes totaux consommés en 2015 sont supérieurs à ceux de 1970, néanmoins, en 45 ans, la part de la viande bovine (bœuf et veau) baisse de façon importante, passant de $39 \%$ des volumes de viande consommés en 1970 à seulement $27 \%$ en 2015 , tandis que la viande de volaille bénéficie d'une forte augmentation, progressant de 16 à $31 \%$ des volumes. Sur cette période, les parts des viandes d'ovins-caprins et de porcs restent globalement stables. Pour les produits laitiers, sur la période 1975-1995, la consommation de laits écrémés et demi-écrémés se substitue presque complètement à celle de lait entier (Nichèle et al., 2005), alors que la consommation de fromages croit de près de $30 \%$, que celle des yaourts est multipliée par deux et celle des desserts lactés par près de six (données Insee et Panel Secodip in Nichèle et al., 2005). En revanche, depuis 1998, la consommation des laits liquides diminue. C'est plus récemment, depuis la crise de 2009, que celle des produits laitiers ultra-frais (yaourts et desserts lactés) s'affaisse. Quant aux consommations de fromages et de matière grasse laitière, elles stagnent (tableau 2) voire progressent légèrement sur les dix dernières années.

Des facteurs économiques et sociétaux peuvent expliquer ces variations de long terme (cf. la synthèse

Tableau 1. Évolution de la consommation française des produits carnés (France AgriMer, 2010 ; France AgriMer, 2016a).

\begin{tabular}{|l|c|c|c|c|c|}
\hline $\begin{array}{c}\text { Consommation de viande } \\
\text { (en kg équivalent carcasse } \\
\text { par personne et par an) }\end{array}$ & $\mathbf{1 9 7 0}$ & $\mathbf{1 9 9 0}$ & $\mathbf{2 0 0 0}$ & $\mathbf{2 0 1 0}$ & $\mathbf{2 0 1 5}$ \\
\hline Bovine & 30,0 & 29,7 & 25,8 & 26,0 & 23,5 \\
\hline Ovine (et caprine) & 3,0 & 5,5 & 5,1 & 3,4 & 2,9 \\
\hline Porc & 30,7 & 35,4 & 36,2 & 33,4 & 32,9 \\
\hline Volaille & 12,1 & 22,0 & 24,8 & 24,5 & 26,6 \\
\hline Total produits carnés & 77,6 & 93,7 & 92,4 & 87,5 & 86,1 \\
\hline
\end{tabular}

Tableau 2. Évolution de la consommation française de produits laitiers (GEB-Idele, d'après CNIEL et SS).

\begin{tabular}{|l|c|c|c|c|}
\hline \multirow{2}{*}{ Consommation de produits laitiers } & \multicolumn{2}{|c|}{ Total (1 000 t) } & \multicolumn{2}{c|}{ En kg/habitant } \\
\cline { 2 - 5 } & $\mathbf{2 0 0 5}$ & $\mathbf{2 0 1 5}$ & $\mathbf{2 0 0 5}$ & $\mathbf{2 0 1 5}$ \\
\hline Lait de consommation & 3200 & 3268 & 54,3 & 49,2 \\
\hline Yaourts et desserts lactés & 1964 & 1936 & 33,3 & 29,2 \\
\hline Fromages (tous laits) & 1468 & 1571 & 24,9 & 23,7 \\
\hline Beurre & 480 & 532 & 8,1 & 8,0 \\
\hline
\end{tabular}

de Delanoue et Roguet (2015) sur les controverses adressées à l'élevage et celle de Legendre et al. (2017) traitant des controverses sur la consommation de viande). Ainsi, la croissance des quantités consommées entre 1970 et 1999 est concomitante d'une augmentation des revenus et du pouvoir d'achat moyen des ménages, ceci se traduisant par une élévation de la consommation journalière de protéines et par une substitution des protéines d'origine végétale par celles issues d'animaux. Pour les produits carnés, les prix relatifs jouent, encore aujourd'hui, un rôle majeur dans l'explication des évolutions de consommation inter-espèces ou intra-espèce. Les prix moyens diffèrent significativement entre espèces $(15,8 € / \mathrm{kg}$ pour la viande fraîche de veau contre $7,3 € / \mathrm{kg}$ pour la viande porcine fraîche), mais aussi entre produits issus d'une même espèce $(10,5 € / \mathrm{kg}$ pour la viande hachée fraîche contre 14,4€/kg pour la viande de bœuf piécée fraîche) (données du "Kantar World Panel», in France AgriMer, 2015a). À partir de 2008, la crise économique a contribué à amplifier la part de marché des produits carnés les moins chers, particulièrement la viande hachée surgelée et certaines viandes de volailles (France AgriMer, 2015b). La consommation de viande, et en particulier celle de viande de bœuf et de porc, jadis supérieure dans les catégories sociales favorisées est désormais plus importante dans le bas de l'échelle sociale (Laisney, 2013).

Les préférences des ménages changent aussi sous l'effet des évolutions de la société et des modes de vie (développement de l'emploi des femmes, urbanisation croissante, pause méridienne sur le lieu de travail...). Ces évolutions se traduisent par la demande de produits à plus longue durée de vie, pouvant être préparés rapidement et consommés en tous lieux. La praticité est plébiscitée. En cinquante ans, l'augmentation de la consommation de produits élaborés, très transformés et marquetés, est considérable (plats préparés à base de viande, desserts lactés) au détriment des produits moins transformés (Agreste, 2017). Ainsi, à titre d'exemple, les consommations nationales de fromages de chèvre et de brebis n'ont que très légèrement augmenté en dix ans 
(respectivement $+5,4$ et $+2,1 \%$ ) (France AgriMer, 2016b), alors que celles des produits frais et ultra-frais des mêmes laits sont actuellement en pleine expansion. De même, les morceaux entiers à bouillir et à braiser de viande bovine, utilisés pour l'élaboration de bœuf bourguignon ou de pot de feu, se vendent mal en dépit d'un prix relatif favorable, alors que les steaks hachés sont passés de $25 \%$ (1995) à $42 \%$ de la consommation de bœuf des ménages (France AgriMer, 2012). A contrario, la viande ovine est pénalisée par le peu de produits transformés et une découpe qui reste « basique».

Il est à noter qu'une part de la consommation nationale se fait sous la forme d'ingrédients incorporés peu visibles dans les plats cuisinés. Pour le secteur laitier, c'est $33 \%$ de la consommation qui passe par les industries agro-alimentaires à travers les activités de biscuiterie, I'alimentation infantile, etc. Ces formes de demande génèrent d'importants échanges entre opérateurs et structurent une partie des importations (Perrot et al., 2016).

En France, ce sont surtout des raisons hédoniques, festives, symboliques qui président à la consommation des produits animaux (Birlouez, 2012 ; Brulotte et Di Giovine, 2016). Ainsi, les produits laitiers et notamment le fromage sont historiquement considérés en France comme structurant du repas (Roberts et Micken, 1996). Néanmoins, les différentes crises sanitaires ou fraudes au cours des deux dernières décennies ont érodé la confiance de certains consommateurs dans les produits d'origine animale (Setbon et al., 2005). De plus, les controverses sur l'impact négatif de la consommation de produits animaux sur la santé (cancer, maladies cardiovasculaires, allergies alimentaires...) se développent depuis les années 1980 (Combris et al., 2011). Jusqu'alors limitées essentiellement à la viande, elles concernent aujourd'hui également le lait (Norat et Riboli, 2003 ; Michaëlsson et al., 2014).

Sous l'influence de l'ensemble de ces dimensions, la sensibilité des consommateurs à des critères de qualité distinctive (organoleptiques, nutritionnels ou mettant en avant les modes de production, un terroir ou un patrimoine) augmente (Tavoularis et al., 2007 ; Verbeke et al., 2010). Plus récemment, d'autres critères de qualité distinctive apparaissent sous l'influence des nouvelles préoccupations sociétales, tels que l'impact des activités d'élevage sur l'environnement, le respect du bienêtre animal, ou les interrogations sur le droit à élever et abattre un animal pour le consommer. Les travaux d'analyse de la perception qu'ont les consommateurs des produits, de leurs appréciations sur l'étiquetage, de l'évolution de leur consentement à payer mettent en évidence l'augmentation de leur sensibilité à une diversité de critères de qualité distinctive (Grunert et Valli, 2001 ; Roosen et al., 2003 ; Chameroy, 2013). Néanmoins, le consentement à payer des consommateurs n'est pas toujours suffisamment important (voir l'analyse en viande ovine de Font i Furnols et al., 2011). Par ailleurs, la consommation effective de produits animaux sous signe de qualité est relativement stable depuis 10 ans et ce, tous produits confondus (données Inao, CNAOL, ODG, Agreste Enquête annuelle laitière, ODG, Synalaf, Sylaporc, Filrouge, in Agreste 2017). Ainsi, pour Harper et Henson (2001), le fait que cette augmentation de la sensibilité des consommateurs ne se traduise pas systématiquement par un achat révèle l'existence de nombreux obstacles - manque d'information, manque de disponibilité, manque de confiance sur sa propre capacité d'influence, dissociation entre bassin de production et de consommation, coût - qui empêchent les consommateurs d'exercer leur préférence éthique dans le choix des aliments.

\section{Des évolutions importantes des politiques publiques et de la réglementation}

\subsection{Du soutien}

de la production

au démantèlement

des outils d'intervention: vers une libéralisation des marchés

En cinquante ans, c'est un véritable changement de paradigme dans les mécanismes de régulation que connaissent les marchés agricoles. L'administration des prix, instaurée dès les années 1960, fait aujourd'hui place au laisser-faire des marchés et à la gestion des conséquences des instabilités de prix. En effet, au sortir de la seconde guerre mondiale, des outils ont été mis en place pour protéger le marché européen (droits de douane) et stabiliser les prix (mesures d'intervention, de stockage et d'écoulement des produits). Leur démantèlement et/ou leur assouplissement progressifs interviennent dès les années 1980 pour la viande ovine (Nozières, 2014), puis dans les années 1990 pour la viande bovine, et à partir de 2000 pour le lait, jusqu'en 2015 avec la suppression des quotas (Trouvé et al., 2016). Pour réduire les coûts et se mettre en conformité avec le droit du commerce international, les droits de douane sont progressivement réduits, ainsi que les prix d'intervention qui aujourd'hui s'apparentent à un filet de sécurité. Initialement administré à un niveau satisfaisant pour les producteurs et les consommateurs, les prix européens se sont progressivement rapprochés des cours mondiaux. Ceci s'est aussi traduit par une augmentation de leur volatilité. Ces changements marquent tous les secteurs (Perrot et al., 2016), de manière inégale du fait d'un pas de temps spécifique des réformes, de l'internationalisation plus ou moins marquée des opérateurs et de niveaux de compétitivité externe différents. Pour compenser ces effets, la PAC s'est concentrée sur des aides directes liées à la production puis découplées et associées à un second pilier axé sur le développement rural, contribuant à une stabilité des revenus, aujourd'hui de moins en moins assurée (Chatellier et Jacquerie, 2004).

Le marché français du lait est emblématique de ces transformations. À partir de 1984, la régulation des marchés laitiers européens a été étendue de l'administration des prix à celle des volumes. En France, le choix de mettre en œuvre des quotas laitiers non marchands, liés au foncier et gérés à l'échelle départementale, leur a conféré une dimension institutionnelle et a permis aux producteurs d'exercer un contrôle collectif sur leur devenir (Dervillé et al., 2012). Les laboratoires 
interprofessionnels ont organisé le paiement du lait à la qualité. Ainsi, jusqu'en 2008, l'accès au marché des éleveurs laitiers était négocié, dans le cadre d'un marché administré (Dervillé et al., 2016). Mais depuis 2008-2009, le secteur est confronté à un changement de régime de concurrence (Dervillé et Allaire, 2014a) en particulier du fait des transformations dans les modalités de gouvernance de la filière avec une mise sous pression des organisations professionnelles et interprofessionnelles et la création d'organisations de producteurs chargées, dans le cadre du Paquet Lait (2012) puis de l'Organisation Commune de Marché unique (2013), de négocier les conditions contractuelles d'accès au marché. La crise que connaît le secteur depuis 2015 tend à montrer que les dispositifs publics et privés élaborés pour faciliter la coordination de la filière sont à ce jour insuffisants ou insuffisamment appropriés (Trouvé et al., 2016 ; Dervillé et al., 2016).

\subsection{Le développement} des politiques « de qualité » et le poids grandissant de la réglementation sanitaire

Dans les années 1990, les crises sanitaires majeures et très médiatisées (Encéphalopathie Spongiforme Bovine - ESB, dioxine, légionellose...), ainsi que l'évolution des attentes des consommateurs, conduisent à développer ou renforcer les politiques de qualité en France et en Europe. Mais la construction du marché unique européen a fait apparaître des différences de logiques, en matière de qualité et dans la mise en œuvre des normes associées, entre i) une vision anglo-saxonne où la qualité se définit comme un standard se limitant aux aspects sanitaires et nutritionnels et ii) une vision latine où prédomine la valorisation d'un patrimoine, d'une culture, d'un savoir-faire. Depuis les années 1990, un compromis instable articule les deux conceptions, et les controverses restent vives, entre entrave à la concurrence et protection des acteurs (Sylvander, 1996).

Sont encadrés par la réglementation européenne, d'une part, les dispositifs de certification officielle de la qualité par l'origine ( $A B, I G P$ ou $A O P$ ) qui soutiennent le développement agricole, notamment dans les zones difficiles, et, d'autre part, les outils permettant de garantir la sécurité sanitaire des produits alimentaires tout au long des chaînes de production. Ainsi, depuis 1991 et 1992, les règlements européens définissant les dispositifs de certification de la qualité des produits par l'origine n'ont cessé d'évoluer. Après les avancées sur la labellisation des produits de montagne (Règlements UE $1151 / 2012$ et $665 / 2014)$, des discussions sont aujourd'hui en cours sur l'opportunité de labelliser les circuits courts (COM866, 2013).

Sur le plan sanitaire, le règlement européen dit « Paquet hygiène ", adopté en 2002, transfère aux opérateurs, à chaque stade de la filière, agriculteurs compris, la responsabilité juridique de la qualité sanitaire des produits, générant des règles sur la traçabilité des denrées alimentaires et donnant lieu à des innovations (systèmes d'information globaux, logiciels couplant traçabilité et gestion de production...). Plus globalement, les réglementations sanitaires induisent des recompositions au sein des filières, liées notamment au coût important des mises aux normes, entrainant des fermetures et/ ou des rachats de structures. Ces réglementations peuvent aussi influencer les échanges internationaux ainsi que les stratégies d'internationalisation des entreprises, en jouant comme des barrières non tarifaires aux échanges (Pion, 2016).

\section{Des opérateurs de l'aval qui se recomposent}

La globalisation et la libéralisation des échanges, associées à la maturité des marchés intérieurs, ont pour effet un accroissement de la concurrence entre entreprises (Rastoin, 2000) et entre territoires, aux échelles mondiales, européennes et locales. De ce fait, l'ensemble des acteurs portent une attention accrue à leurs marges globales (Rastoin, 2008). Ils se concentrent (cf. $\S 3.1$ et 3.2 ) et cherchent de nouveaux marchés, à
I'international (cf. § 3.3) ou travaillent, souvent avec les éleveurs, l'offre à destination des consommateurs (cf. § 5).

\subsection{Développement et concentration des opérateurs de collecte et de transformation}

Si la période 1970-1980 a été caractérisée par une phase de concentration industrielle et d'amélioration de la productivité, c'est depuis les années 1990 que les nombreuses restructurations financières engendrent une forte concentration des structures de collecte et de transformation (Rastoin, 2000). À noter que cette tendance touche aussi bien les entreprises de droit commercial que le secteur coopératif (Filippi et Triboulet, 2011). Cette concentration des industries laitières (Ricard, 2014) et de la viande (De Fontguyon et Sans, 2003) pose la question de l'équilibre concurrentiel des marchés. Elle a, en effet, conduit à des structures de marchés de type « oligopoles à frange » dans lesquelles quelques gros opérateurs dominent, cohabitant avec de nombreuses petites structures (voir pour le secteur viande bovine l'article de Soufflet 1990). Néanmoins, le degré de concentration varie selon les produits concernés. Par exemple, alors que pour le beurre et le lait liquide, les trois premiers groupes commercialisent respectivement 59 et $69 \%$ des fabrications nationales, pour le fromage, les vingt premiers établissements traitent $45 \%$ seulement de la production nationale (France AgriMer, 2016b).

Le processus de concentration interroge aussi le maintien des outils de transformation dans les territoires de production. Ainsi, si la capacité d'abattage à l'échelle nationale reste importante, le maillage territorial laisse apparaître des zones peu dotées en outils industriels. La disparition de ces outils s'accompagne d'une perte de savoir-faire et réinterroge la possibilité du maintien de la valeur sur ces territoires. Cette situation conduit, dans certains cas, la puissance publique à soutenir certains d'entre eux en dépit de leur non rentabilité économique (Guiomar, 2011). 


\subsection{Des distributeurs} très concentrés appliquant une stratégie de domination par les coûts

Depuis leur émergence à la fin des années 1950, les opérateurs de la grande distribution n'ont cessé de se développer pour devenir aujourd'hui des acteurs majeurs des filières. Bénéficiant de l'augmentation du pouvoir d'achat des ménages, l'expansion de ce modèle de distribution de masse a accompagné le développement de l'industrie agroalimentaire. Dès les années 1970, la croissance de la consommation commence à ralentir, l'environnement réglementaire et concurrentiel se durcit (Daumas, 2006), ce qui entraine des fusions-acquisitions des enseignes, qui s'accélèrent au cours des années 1990. Actuellement, en France, six grands groupes internationaux représentent près de $90 \%$ des parts de marché de la grande distribution, avec, récemment, une accélération des stratégies d'alliance entre groupes pour le regroupement des achats. Aujourd'hui, les grandes surfaces d'alimentation générale commercialisent près des deux tiers des ventes de produits alimentaires hors tabac (INSEE, 2017) et constitue le circuit dominant des ventes de produits carnés et laitiers. On constate, néanmoins, que les commerces alimentaires spécialisés indépendants résistent mieux pour la viande que pour les produits laitiers. En 2000, ils représentaient une part de marché de $25 \%$ pour la viande, contre $3 \%$ pour les produits laitiers (de Kermadec, 2011).

La domination par les coûts reste la stratégie de base pour le développement de ces grandes enseignes (Mevel, 2010). Elle conduit au renforcement de leur puissance d'achat et à l'instauration d'un rapport de force conflictuel, en leur faveur, avec les acteurs de l'amont (Allain et Chambolle, 2003). Dans les années 1990, la nature des relations entre ces acteurs et leurs fournisseurs connait une inflexion. Sous la pression croissante de leurs concurrents directs et des grands groupes de l'industrie et en réponse à l'évolution profonde des modes de consommation, les distributeurs développent leurs marques propres (Lepers, 2003).
Alors que cette stratégie s'inscrit, dans un premier temps, dans un objectif de compétitivité prix avec des marques de distributeur premier prix, elle répond par la suite à un objectif de gain de compétitivité hors prix, avec le développement de marques de distributeur "premium » qui s'appuient sur des cahiers des charges spécifiques (marques de distributeur "terroir», marques de filière) (cf. § 5).

\subsection{Des stratégies} d'opérateurs de plus en plus structurées par le poids des échanges mondiaux

Tout en se concentrant, les opérateurs d'aval recherchent de nouveaux vecteurs de croissance. Le marché domestique des produits carnés et laitiers étant mature ou quasi-mature, l'export au sein de l'Union européenne mais surtout vers les pays-tiers, peut présenter des opportunités. Ainsi pour le lait de vache, la demande internationale, bien qu'en croissance modérée et fluctuante (Trouvé et al., 2016) constitue aujourd'hui le moteur principal du développement des opérateurs concernés, avec un appel sous forme essentiellement de fromages et de laits secs, émanant en particulier de la Chine, mais aussi du Royaume-Uni et de l'Italie (Perrot et al., 2016). En viande bovine, le décalage entre la nature de l'offre nationale et celle de la demande (type d'animaux, caractéristiques de viandes) conduit certains opérateurs à se spécialiser sur des marchés d'export (Idele, 2016b). Soixante pour cent des quantités de viande ovine consommées sont importées (Idele, 2016a), ce qui structure fortement la stratégie des transformateurs et des distributeurs.

\subsection{Des modes \\ de coordination \\ entre opérateurs \\ qui évoluent}

Le développement de contrats entre opérateurs, voire l'intégration entre structures, et la perte de vitesse progressive des formes d'organisation moins centralisées et formalisées (transactions de gré à gré, marchés physiques) traduit une évolution forte des modes de coordination au sein des filières agro-alimentaires vers des dispositifs de plus en plus formalisés. Réseaux de sous-traitance, réseaux d'entreprises, partenariat, alliance, etc., les formes organisationnelles hybrides, associant entre-elles les structures d'aval, se développent (Ménard, 2003). La gouvernance des filières animales évolue donc vers des formes de plus en plus centralisées.

Les contrats coordonnent les activités des opérateurs en définissant, de façon formelle, le type de bien ou de service échangé, la quantité et les qualités attendues, le processus de production, la rémunération, la fréquence des échanges et les investissements à réaliser. Ces modalités de coordination sont destinées autant à maîtriser la concurrence qu'à sécuriser les relations avec les fournisseurs et fiabiliser l'approvisionnement, en termes de quantité mais surtout de qualité. Le développement de ces contrats a été porté non seulement par les stratégies des acteurs privés mais également par les politiques publiques (cf. § 4.2). II a été renforcé par la progression des stratégies de segmentation de l'offre pour le consommateur qui s'appuient sur la définition de cahiers des charges spécifiques (cf. § 5) (Sans et Coquart, 1998).

Certaines stratégies de concentration industrielle (cf. $\S 3.1$ ) ont conduit à des opérations de filialisation, en particulier dans le secteur laitier, ainsi qu'à des opérations de croissance externe (fusions, acquisitions) (Torre et al., 2013). Elles ont eu pour conséquence une diminution de la division du travail tout au long des filières et à un rassemblement des activités au sein d'entreprises industrielles ayant un poids de plus en plus important. Néanmoins, I'organisation de la collecte et de la transformation des produits carnés et laitiers diffèrent. Pour le lait, du fait de sa nature périssable, relativement homogène à la sortie de la ferme et dont la séparation des constituants donne lieu à une multiplicité de produits, collecte et transformation sont aujourd'hui souvent effectuées au sein d'une même structure. En revanche, dans les secteurs des viandes de ruminants ces opérations sont généralement prises en charge par des acteurs distincts. 
Bien qu'ils ne soient pas majoritaires, certains opérateurs de la distribution ont également adopté des stratégies d'intégration pour soutenir le développement de leurs marques propres. C'est le cas notamment des enseignes Leclerc et Intermarché dans les filières viande. Ainsi, les abattoirs Gilles ont été le support de la création de Kermené (transformation de produits carnés), par l'enseigne Leclerc en 1978. L'enseigne Intermarché, quant à elle, mène une stratégie d'intégration de ses fournisseurs avec le rachat de la laiterie SaintPère en Retz en 1990 et de l'abattoir SVA Jean Rozé en 2001, les adhérents de ce réseau s'affichant aujourd'hui comme des « producteurs commerçants ».

Ainsi, c'est un véritable changement de paradigme auquel sont soumises les filières de produits carnés et laitiers, puisqu'il ne s'agit plus de penser «de la fourche à la fourchette », mais bien "de l'assiette au champs» (cf. § 1). De plus, l'environnement dans lequel agissent les opérateurs des filières est devenu très concurrentiel, générant de profondes recompositions (cf. § 2 et 3). Le modèle mis en place depuis les années 1970, organisé autour de flux de produits standardisés incite à la permanence d'un avantage coût, notamment via des gains de productivité (Mevel, 2010). La captation par les producteurs d'une part significative de la valeur, suffisante pour couvrir les coûts de production et dégager un revenu, est alors en question. Ceci interroge tout autant l'efficacité de leurs organisations collectives et de leurs modes de coordination avec les autres opérateurs (cf. $§ 4$ ), que leur capacité à peser sur la segmentation de l'offre au consommateur et leur autonomie dans les choix de modes de production (cf. § 5).

\section{Gouvernance et structuration des filières : quel poids des éleveurs ?}

Encadrés par les politiques publiques, les outils pour la gouvernance et la structuration des filières ont fortement évolué dans les dix dernières années. Ils ont aujourd'hui pour fonction centrale de pallier les limites de la coordination par le seul marché tout en respectant le cadre dessiné par le droit à la concurrence. De ce fait, un glissement s'opère d'une régulation publique du marché à la mobilisation d'outils privés.

\subsection{Des interprofessions fragilisées}

Constituées à l'initiative de leurs membres, les organisations interprofessionnelles sont des institutions de droit privé recevant une délégation de pouvoir de l'administration. Réunissant les différents maillons d'une filière, elles ont été reconnues en France vers 1970 autour de compétences de médiation entre opérateurs, de production d'information économique, de représentation et de gestion des questions relatives à la qualité. Elles jouent ainsi un rôle majeur dans l'organisation des relations au sein des filières et participent en particulier à la mise en place de contrats entre opérateurs et entre les éleveurs et les premiers acheteurs. Bien que de telles structures de gouvernance existent aux États-Unis ou au Canada, elles constituent une spécificité française au sein de l'union européenne et ont été reconnues par Bruxelles seulement en 2013 (Cadilhon et Dedieu, 2011).

Les laboratoires interprofessionnels et l'interprofession laitière (CNIEL) rassemblent des représentants des fédérations des producteurs de lait, des coopératives et des industries. Ils sont le lieu d'élaboration collective de la qualité du lait. De 1997 à 2008, le CNIEL a également été le lieu de négociation du prix du lait. L'interprofession de la filière bétail et viande (INTERBEV) rassemble 21 organisations nationales dont les distributeurs. Cette extension à la distribution s'est faite pour assurer une réaction coordonnée aux crises de l'ESB, notamment via la création de deux marques collectives "Viande Bovine Française » et «Critères Qualité Contrôlés » (Sans et De Fontguyon, 1999). D'autres interprofessions comptant un petit nombre d'acteurs sont structurées autour de la valorisation d'un produit, par exemple sous Signe d'Identification de la Qualité et de l'Origine (cf. sur le Comté l'article de Rigolot, 2016).
Il semble que ces instances interprofessionnelles, hormis celles structurées autour d'AOP, peinent aujourd'hui à trouver des solutions collectives pour gérer les risques et garantir un partage équitable de la valeur dans un environnement instable et globalisé (Allain et Chambolle, 2003 ; Trouvé et al., 2016). Le médiateur des relations commerciales agricoles, fonction créée en 2010 au sein du Ministère de l'Agriculture et de I'Agro-alimentaire peut ainsi être saisi pour régler les litiges liés aux relations contractuelles, ou simplement pour donner un avis, prenant ainsi partiellement le relai des interprofessions. Quoiqu'il en soit, la complexité de la construction des marges à chaque maillon de la filière et l'évolution rapide de leur répartition entre opérateurs (Boyer et al., 2013) rendent complexes les négociations entre acteurs. Bien que le statut des différentes professions au sein des interprofessions, avec des organisations professionnelles représentatives, leur garantisse théoriquement un poids égal dans les décisions, le fonctionnement de ces instances traduit souvent le déséquilibre des rapports de force, en particulier entre la production et les premiers acheteurs. Ainsi, se pose la question du renforcement en leur sein, du maillon "producteurs». La structuration des relations entre producteurs et premiers acheteurs, d'une part, et l'organisation des producteurs entre eux, d'autre part constituent des enjeux importants (Cadilhon et Dedieu, 2011).

\subsection{Des contrats entre producteurs et collecteurs pour sécuriser les débouchés?}

Dans un marché libéralisé, les politiques européennes incitent à la contractualisation entre les producteurs et leurs collecteurs et ce, dans les secteurs des viandes (Marty et al., 2015) comme du lait (Berger et al., 2015). Ces outils sont vus comme un moyen de sécuriser les débouchés des producteurs et de rééquilibrer les pouvoirs de négociation, notamment dans le cadre du démantèlement des outils de régulation des marchés (cf. § 2). Néanmoins, si la contractualisation peut contribuer à gérer les risques « prix » et « demande » 
ainsi que ceux induits par les processus de production, mais aussi inciter à la performance quantitative et qualitative (développement de standards de la qualité...), elle ne contribue à modifier l'équilibre des pouvoirs que si les producteurs s'organisent efficacement entre eux (Veerman et al., 2016). L'efficacité d'un contrat dépend beaucoup du contenu et des modalités de son établissement, de la structure du marché (Bouamra-Mechemache et al., 2015), mais aussi de la spécificité du produit et du cadre institutionnel (Trouvé et al., 2016 ; Dervillé et al., 2016). La crise que connaît le secteur laitier depuis 2015 rend compte de l'incapacité des seuls contrats à gérer les crises, à protéger les exploitations dans leur diversité (Berger et al., 2015 ; Trouvé et al., 2016), et ce, d'autant plus lorsque les producteurs doivent négocier individuellement avec leurs acheteurs les conditions de commercialisation de leur produit (Berger et al., 2015 ; You, 2015). En parallèle, l'existence de marchés physiques (visibles essentiellement dans le secteur des produits carnés) où se rencontrent fournisseurs et acheteurs et sur lesquels les gestionnaires de marché, encadrés par l'État, prennent en charge la publication de cotations (cf. le Réseau des nouvelles du marché, service de France AgriMer) introduit de la transparence dans des marchés de plus en plus libéralisés, condition importante pour le déroulement de transactions (Cinquegrana, 2008). Plus largement, l'accès à l'information et sa valorisation sont un enjeu pour les opérateurs et engagent la responsabilité de l'État.

\subsection{S'organiser entre} éleveurs pour avoir plus de poids dans les échanges?

L'organisation collective pour la commercialisation (et la transformation) est une possibilité pour peser dans les négociations avec les opérateurs d'aval, en matière de volume et de prix et ainsi rééquilibrer le rapport de force au sein des filières.

Ainsi, les Organisations de Producteurs (OP) ont pour fonction de regrouper l'offre de production et de rééquilibrer ainsi les relations commerciales. Ces OP, qui peuvent avoir des statuts variés (coopératives, associations, sociétés commerciales), doivent être reconnues par l'État. Les OP commerciales collectent la production de leurs adhérents et prennent en charge sa commercialisation. Les OP non-commerciales fournissent à leurs membres les moyens, humains, matériels ou techniques, pour assurer la commercialisation de leur production. L'existence d'une OP conduit souvent les producteurs à " parler prix », et la frontière avec une entente sur les prix, considérée par les autorités européennes comme une atteinte au droit de la concurrence, est toujours floue. Néanmoins, par exemple, dans la situation actuelle du secteur laitier, le « Paquet Lait » de 2012 encourage la formation d'OP ou d'associations entre OP jusqu'à $33 \%$ de la collecte nationale et donne aux producteurs la possibilité de négocier collectivement le prix du lait sans transfert de propriété. Le renforcement du pouvoir de négociation des OP favorisant la transparence des informations, le respect des engagements, la définition du contenu et des modalités d'établissement des contrats, pourrait modifier sensiblement l'équilibre du marché au profit des producteurs (Berger et al., 2015 ; Trouvé et al., 2016).

La coopérative, quant à elle, en tant que structure collective de gestion de la collecte et, pour le secteur laitier, de transformation, joue un rôle central dans la sécurisation de l'accès au marché et la gestion des risques mais aussi dans le partage de la valeur ajoutée. Elle a également une utilité dans la maîtrise des ressources, logistiques mais aussi informationnelles et communicationnelles. Mais elle présente des fragilités. Mainsant (2012) souligne la faiblesse des capitaux propres liés au fonctionnement coopératif ainsi que le poids des compétences issues du militantisme syndical qui rend difficile la gestion commerciale et l'action en situation ultra-concurrentielle, en particulier le travail sur la compétitivité. De plus, la place des éleveurs dans les prises de décision au sein de coopératives grossissantes (et de plus en plus intégrées dans des groupes) est en question. Ce processus n'est pas spécifique aux secteurs carnés et laitiers et globalement, les relations adhérents-coopératives se distendent (Barraud-Didier et al., 2012) et se complexifient (Hansen et al., 2002 ; Cechin et al., 2013) Dans le secteur de la viande bovine où la concurrence entre coopératives et entreprises de droit privé a toujours été rude, et où la règle de l'apport total est difficile à faire respecter, les éleveurs finissent par se désengager de structures de grande taille, qui s'en trouvent fragilisées. Néanmoins, d'autres stratégies semblent possibles, comme celles portées par des groupes coopératifs très ancrés régionalement (Arcadie SudOuest, Sicavyl...) (Mainsant, 2012). Dans le secteur laitier, le verrouillage de la collecte par les acteurs historiques n'empêche pas le développement d'autres modèles de fonctionnement, au statut privé, mais adoptant des valeurs coopératives de transparence et de solidarité (voir l'exemple de Biolait dans Chiron, 2015).

Les stratégies des concurrents non coopératifs (Danone, Lactalis, Bigard...) incitent les coopératives à les imiter : intégration verticale, internationalisation, diversification, (Filippi et al., 2008). Ainsi des groupes coopératifs se constituent : union pour gérer des marques (Sodiaal), groupes pour assurer la transformation et dont la commercialisation est l'activité dominante (Socopa), groupes polyvalents (Terrena). Les activités se complexifient et la polyvalence se développe. La création de filiales de droit commercial le plus souvent codétenues par les coopératives s'amplifie également. Ce mouvement interroge l'exercice du droit de propriété des agriculteurs adhérents (Galliano, 1995 ; Triboulet et Filippi., 2013). Dans le secteur laitier, les coopératives ont développé des filiales par produits, pouvant aujourd'hui être codétenues par plusieurs entreprises de statut différent. Dans le secteur de la viande ce processus a conduit à la privatisation complète de la transformation. En effet, si, dans les années 1970, une partie des acteurs de la collecte de bovins avaient fait le choix d'entrer au capital de structures industrielles telles que Socopa ou Arcadie, un démantèlement de ces outils coopératifs s'est opéré à partir de 1986, au profit de la montée en puissance de Bigard, commercialisant près de $40 \%$ des bovins abattus en France (Mainsant, 2012). 
Aujourd'hui, dans les secteurs de la viande, les liens entre structures de collecte et de transformation, s'appuient sur des relations de marché, éventuellement sur des contrats, plus que sur des opérations de filialisation ou d'intégration, à quelques exceptions près, par exemple l'Agneau Soleil et l'ancien groupe Dufour à Sisteron (Nozières et al., 2015).

D'autres formes de regroupement d'éleveurs peuvent exister pour la commercialisation de leurs produits, en particulier des formes associatives, par exemple l'initiative autour de la marque "l'éleveur et l'oiseau » (Billaudeau et Thareau, 2010). Dans certains cas, de nouveaux acteurs s'engagent pour contribuer à structurer une filière, tels que des collectivités territoriales (Capt et al., 2014). Plus globalement, ces différentes modalités de structuration des filières et en particulier du lien entre les éleveurs et leurs premiers acheteurs, dépendent, entre autres, des besoins identifiés pour accompagner le développement de stratégies de segmentation de l'offre pour le consommateur.

\section{La segmentation de l'offre pour le consommateur, l'affaire de tous les acteurs des filières?}

\subsection{Une segmentation} de l'offre portée par les opérateurs de l'aval

Après une phase d'industrialisation des « process » dans les années 19501960, et une période de diversification dans les années 1970-1980 (Rastoin, 2000), depuis les années 1990 les industries de transformation des produits laitiers et carnés recentrent leurs activités et améliorent leurs « process » pour maîtriser la qualité. L'innovation est leur maître mot, en viande bovine (Sans, 2003) comme pour les produits laitiers (Henning et al., 2006), ce qui nécessite de s'engager dans des investissements immatériels (Coquart et Soufflet, 1989). Les opérateurs des filières portant une attention accrue aux attentes qualitatives des ménages (Grunert et Valli, 2001), un réel dynamisme est généré dans les champs de la recherche technologique, conduisant à proposer aux consommateurs un certain nombre de produits innovants. Le champ du marketing se développent largement (cf. par exemple Ares et Deliza, 2010). Le dynamisme en matière d'innovation et de marketing dépend toutefois de la nature du produit, et en particulier de son niveau de différentiation du départ (Grunet et Valli, 2001), ce qui induit un contraste entre les produits carnés et laitiers (Soufflet, 1990). Ces innovations sont souvent « de bout de chaîne » créées à partir de matière première standard, coûteuse en compétences mais ne favorisant pas ou peu le retour de valeur en amont.

Portés par les distributeurs, les stratégies de marques de distributeurs ou de filières sont souvent fondées sur le développement de relations contractuelles bilatérales avec des PME en charge de la transformation, ou tripartites avec un industriel et des éleveurs. Au-delà des attentes en termes de typicité de produits, ces marques ont permis aux distributeurs dans le secteur de la viande bovine de répondre aux préoccupations fortes de consommateurs en matière de sécurité sanitaire suite aux crises de l'ESB (De Fontguyon et al., 2003). Néanmoins, avec ces outils, la grande distribution alimentaire dépasse aujourd'hui sa fonction traditionnelle, devenant prescriptrice des conditions de production (Burch et al., 2013) en participant à la définition des produits au niveau de la transformation comme de la production agricole (Baritaux et Billion, 2016). Ces démarches apportent une dimension relationnelle aux échanges et sont souvent mises en avant puisque, localement, elles peuvent permettre un rééquilibrage des rapports de force et un meilleur partage de la valeur (Baritaux et Houdart, 2015). Néanmoins, les questions sur la nature réelle des relations avec les fournisseurs sont nombreuses, ces marques pouvant être vues également comme un moyen de faire des distributeurs de véritables « pilotes » des systèmes alimentaires (Bergès-Sennou et Caprice, 2003).
5.2. Segmenter l'offre par des Signes d'Identification de la Qualité et de l'Origine (SIQO) pour structurer les filières et assurer une meilleure valorisation des produits pour les éleveurs

Le développement d'une demande de produits de qualité faisant référence à des attributs intangibles des produits, qui intègrent les modes de production, appuyée par la présence de politiques de développement de la qualité peut contribuer à la création de ressources spécifiques. La valeur de ces ressources est liée au processus productif qui les a fait naître, ce qui devrait favoriser le partage équitable de la valeur créée entre les différents opérateurs de la filière.

Ces démarches de qualité ont souvent été construites à l'initiative des producteurs généralement organisés entre eux, par exemple dans le cas du Pélardon (Boutonnet et al., 2005), ou de la viande de Maine Anjou (Noury et al., 2005). Ces éleveurs souhaitaient accroître leur autonomie, sécuriser l'écoulement de leur production (Nozières, 2014 ; Dervillé et Allaire, 2014a) et faciliter la captation d'une plus grande part de la valeur. Ces démarches s'appuient sur la typicité des produits et la valorisation de savoir-faire locaux (AOP, IGP), soutenant ainsi le développement des territoires, comme par exemple dans le cas du Comté (Fumey et Bérion, 2010 ; Dervillé et Allaire, 2014a), et mobilisant comme une ressource, le territoire à travers l'action collective (Chabrat, 2015). Certains certifient une qualité supérieure (Label Rouge), le choix de modes de production garantissant un respect de l'environnement $(A B)$ ou une typicité des produits (AOP, IGP). Au sein de ces démarches, la diversité des systèmes d'élevage peut être vue comme un gage de capacité d'adaptation et une garantie de la typicité de certains produits (Perrot et al., 2005).

Cette segmentation a contribué à structurer verticalement les différentes filières et à orienter les stratégies des opérateurs. Ainsi de la forte mobilisation de la grande distribution sur le segment de I'Agriculture Biologique (AB), 
comme celle de l'enseigne Auchan, initiée par un accord conclu avec la FNAB pour la viande bovine dès 1996. De même, l'enseigne Carrefour, après avoir développé une gamme Bio, déploie depuis 2016, une enseigne "Carrefour Bio » pour les centres villes. Ces différentes actions soutiennent en retour le développement des élevages en $A B$, ce qui illustre à nouveau l'influence de la grande distribution sur les modes de production.

En France, l'offre totale de produits laitiers sous SIQO augmente de 161 à 241 milliers de tonnes entre 1991 et 2013 (Agreste, 2015), les fromages AOP représentant $10 \%$ du lait français produit (mais $15 \%$ de la consommation de fromages en volume et $25 \%$ en valeur) et I'AB moins de $3 \%$ de la collecte, mais $10 \%$ du lait liquide conditionné. Aujourd'hui, dans le secteur des viandes, hormis pour celles de volailles, les volumes commercialisés sous SIQO restent faibles : environ $30000 \mathrm{t}$ pour les viandes bovines, 10000 t pour la viande ovine et 47000 t pour la viande porcine en 2014 et progressent peu (Agreste, 2016). Cependant, un dynamisme particulier aux viandes issues de l'AB est à noter sur la dernière décennie.

L'existence d'une segmentation se révèle également très utile pour conquérir des marchés autres que locaux comme c'est le cas du Pélardon (Benkahla et al., 2005). Mais les risques existent, tel que celui de réappropriation par des acteurs globaux, en particulier si le terroir et les caractéristiques du produit ne sont pas assez précisément décrits dans les cahiers des charges et si l'organisation collective et le rôle de l'État ne sont pas bien définis (Barjolle et Sylvander, 2003 ; Bowen, 2010). L'association entre ces logiques d'aval et la valorisation des produits sous SIQO peut occasionner des tensions (Filippi et Triboulet, 2006) : exclusion de producteurs locaux, désaccord sur la nature et la gestion des ressources utiles, injonction à produire toute l'année occasionnant un risque de perte de spécificité (Aubron et al., 2014). Chaque démarche diffère par le niveau d'implication des différents types d'opérateurs, qui varie également au cours du temps, donnant lieu à des compromis souvent instables (Filippi et Triboulet, 2006). Cette grande diversité de formes organisationnelles et institutionnelles conduit à des plus-values très différentes selon les cas (Jeanneaux et al., 2011 ; Dervillé et Allaire, 2014a). De plus, ces SIQO se trouvent fragilisés par le fait que certaines marques de distributeurs et marques de filières s'appuient sur ces notions de typicité et de terroir. C'en est ainsi de l'enseigne Carrefour et de sa marque « Reflets de France » (Beylier et al., 2012) ou des grandes enseignes qui mobilisent des arguments similaires à ceux des SIQO comme la réduction des intrants (filières sans antibiotiques de l'enseigne Carrefour...) ou le respect de l'environnement. Enfin, la multiplicité des signes (SIQO, marques commerciales, collective...) brouille plus qu'elle n'éclaire le consommateur (Tavoularis et al., 2007) et entraîne la dormance de certaines démarches.

\subsection{Segmenter l'offre par les circuits courts (CC) pour redonner du poids à la relation consommateur- producteur}

Pour les produits laitiers, la transformation à la ferme et la vente directe sont des pratiques anciennes. Ainsi en 1958, moins de la moitié du lait était livré à l'industrie (Boichard, 1972). Pour la viande bovine, elles apparaissent, dans les années 1990 au moment où certains éleveurs de moyenne montagne choisissent de créer des boucheries de producteurs et de vendre leur viande en caissette pour faire face à des ventes irrégulières et à des baisses de prix (Michaud, 2015). Depuis, la vente directe n'a cessé de croître sous l'effet des évolutions de la consommation (cf. $\S 1$ ) et de l'organisation des opérateurs des filières (cf. § 3 ).

Si aujourd'hui, $21 \%$ des exploitants français vendent en CC (avec au plus un intermédiaire entre le producteur et le consommateur) (Agreste, 2012), dans les secteurs d'élevage, cette proportion n'est atteinte que pour les caprins et ovins viande (tableau 3), révélant une place limitée de ces circuits. La part du chiffre d'affaires total provenant de la vente en CC varie beaucoup d'une exploitation à une autre, donnant à ce type de circuit un statut inégal, de débouché exclusif à mode de mise en marché anecdotique. Néanmoins, les producteurs laitiers qui vendent en CC en tirent l'essentiel du chiffre d'affaire de l'atelier lait (sauf en bovins lait où la variabilité est grande). C'est encore $54 \%$ du chiffre d'affaire de l'atelier pour les éleveurs ovins viande qui pratiquent les CC mais seulement $37 \%$ pour les ateliers bovins viande (données Agreste RA 2010 - traitement Idele). Pour les éleveurs, les raisons du choix des CC sont souvent multiples, la recherche de plus-value économique restant la motivation première et quasi-unanime. Le souhait d'une meilleure reconnaissance du travail et le goût pour la relation avec les clients interviennent également (Chiffoleau et al., 2013).

Pour les laits, le choix des CC nécessite la prise en charge par l'éleveur de l'essentiel du processus de transformation pour les volumes concernés. Les bovins et ovins viande passent obligatoirement par un abattoir prestataire.

Tableau 3. Part des exploitations agricoles commercialisant en circuits courts, dont vente directe (données Agreste RA 2010 - traitement Idele)

\begin{tabular}{|l|c|c|c|c|c|}
\hline $\begin{array}{l}\text { Type d'exploitations agricoles } \\
\text { (sans double compte) }\end{array}$ & $\begin{array}{c}\text { Bovins } \\
\text { viande }\end{array}$ & $\begin{array}{c}\text { Ovins } \\
\text { viande }\end{array}$ & $\begin{array}{c}\text { Ovins } \\
\text { lait }\end{array}$ & $\begin{array}{c}\text { Bovins } \\
\text { lait }\end{array}$ & Caprins \\
\hline Nombre d'exploitations & 87292 & 11852 & 4879 & 75636 & 6894 \\
\hline $\begin{array}{l}\% \text { avec vente directe } \\
\text { ou circuits courts } \\
\text { pour produits laitiers }\end{array}$ & NS & NS & 16,0 & 4,7 & 42,6 \\
\hline $\begin{array}{l}\% \text { avec vente directe } \\
\text { ou circuits courts } \\
\text { pour produits viande }\end{array}$ & 10,5 & 20,4 & 9,6 & 4,7 & 12,2 \\
\hline
\end{tabular}


Dans près de $60 \%$ des cas, la découpe des carcasses est effectuée par un prestataire de services (Etchevarria, 2013), éventuellement payé «à la tâche » par l'éleveur et travaillant dans un atelier de découpe individuel ou collectif. Cette découpe peut aussi être effectuée par l'éleveur lui-même ou par le distributeur. Aux coûts de prestations s'ajoutent des coûts et des temps de transport, parfois importants du fait du maillage territorial déséquilibré des outils d'abattage.

La distribution en CC peut se faire selon plusieurs modalités. En production fromagère caprine, la vente sur les marchés est le mode dominant dans $39 \%$ des cas (données Agreste RA 2010 - traitement Idele), suivie de près par la vente à la ferme (37\%). Les éleveurs caprins ont aussi souvent recours à un intermédiaire, en particulier les plus gros élevages. Cinquante-quatre pour cent des chèvres sont situées dans un élevage qui utilise un intermédiaire (crémier ou détaillant $35 \%$, grande et moyenne surface $19 \%$, restauration y compris collective $14 \%$ ). En 2010, le poids des magasins de producteurs (4\%) et AMAP (Associations pour le maintien d'une agriculture paysanne) (1\%) apparaissait globalement très faibles. La diversité régionale de ces circuits de distribution est forte: les magasins de producteurs sont surtout présents en Rhône-Alpes et Languedoc-Roussillon, alors qu'en région lyonnaise les marchés s'imposent comme mode de commercialisation dominant (60\% environ). En élevage bovin et ovin, laitiers et allaitants, la vente directe en ferme arrive en tête des modes de distribution. En viande bovine, $28 \%$ des 85 exploitations enquêtées par Etchevarria (2013) n'utilisent qu'une forme de circuits (vente directe à la ferme ou par livraison), qui, pour les autres est associé à plusieurs formes de vente directe (marchés, boutique...). Les grandes exploitations peuvent elles-aussi avoir recours à la transformation et à la vente directe, mobilisant une main-d'œuvre importante par rapport à la moyenne des exploitations. Ainsi, en 2010, 185 exploitations laitières, comparables à des structures artisanales, transformaient du lait en employant plus de
6 UTA : 103 vaches laitières et 9,5 UTA en bovins ou 301 chèvres et 8,3 UTA en caprins (données Agreste RA 2010 - traitement Idele)

Peu de données existent sur la vente en CC de produits sous SIQO. La carte de la figure 1 localisant 6247 exploitations transformant du lait à la ferme en 2010 illustre l'existence de bassins traditionnels de transformation à la ferme, souvent dans le cadre d'une AOP et souvent en montagne. Le maillage régulier du reste du territoire doit beaucoup à des opportunités locales rapidement saturées occasionnant une concurrence entre producteurs. $22 \%$ de ces transformateurs de lait à la ferme sont en $A O P, 11 \%$ en $A B$ (rarement les deux). Le recours à ces SIQO est 3 à 4 fois plus important que pour les non transformateurs, suggérant une synergie entre labels de qualité et circuit court (Dervillé et Wallet, 2014).

Le développement des CC impacte l'organisation globale des filières, et ce, d'autant plus dans les secteurs où l'offre est rare. Ainsi, dans le cas de la viande ovine, vendre en direct peut positionner les éleveurs comme concurrents des bouchers de détail, alors potentiellement fragilisés. Ceci peut aussi apparaître comme un risque pour des OP qui, par ricochet, proposent parfois aux éleveurs une prestation de service pour la transformation. De fait, les hybridations des CC avec les circuits longs se développent dans l'ensemble des secteurs (voir pour le lait, Chazoule et al., 2015) donnant lieu à la création de filières à taille humaine, où la gouvernance collective et la valorisation des métiers tiennent une place importante.

Si l'attention des consommateurs aux modes de production des produits qu'ils achètent ainsi qu'à une plus juste rémunération des producteurs s'exprime, entre autre, par leur intérêt pour les circuits courts, elle est, plus récemment, support de la structuration de la segmentation de l'offre proposée par les distributeurs. C'est ainsi que sont nées récemment des initiatives structurées autour de marques, en particulier pour le lait liquide, comme «C'est qui le patron » ou «Fair France » ou des démarches comme celle «des Alliances Locales»

Figure 1. Localisation des exploitations transformant du lait à la ferme en 2010. Source : Agreste recensement agricole 2010 - traitement Institut de l'élevage

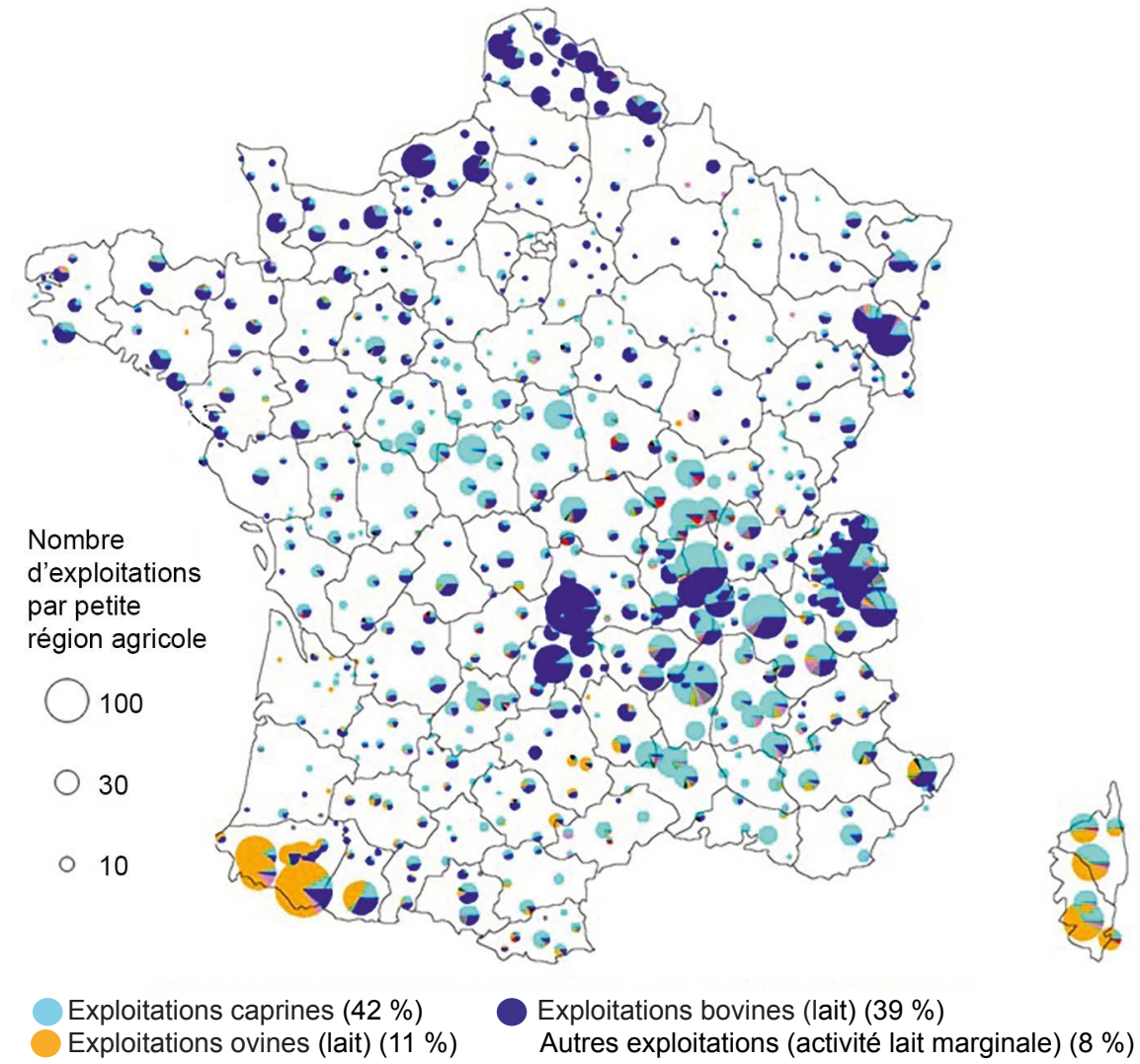

Exploitations ovines (lait) $(11 \%)$
Exploitations bovines (lait) (39\%) Autres exploitations (activité lait marginale) $(8 \%)$ 
portées par Leclerc qui revendique 10500 partenariats entre producteurs et magasins locaux. Dans ces deux cas, le rapprochement du consommateur et du producteur mobilise les canaux de distribution conventionnels (grandes entreprises de transformation et grande distribution). Comme pour les SIQO, le potentiel de valorisation varie d'une démarche à l'autre, entre la stabilisation d'un prix rémunérateur et une simple prime.

Dans ce raccourcissement des circuits (par omission des opérateurs de transformation et de distribution), c'est non seulement le prix payé au producteur qui est en jeu, mais aussi la valorisation du caractère local de la production. Pour ces démarches de différenciation de l'offre, par la certification des produits ou par la production en circuit plus courts, l'ancrage territorial des productions apparait comme un élément clef (Napoléone et al., 2015), la communication et la confiance jouent un rôle essentiel dans la commercialisation des produits (Baritaux et Houdart, 2015). Plus largement, les innovations institutionnelles (cahier des charges, dispositif de gestion de l'offre, clé de répartition de la valeur...) favorisant l'alignement dynamique des intérêts individuels dans un but commun conditionnent le succès de ces démarches (Dervillé et Allaire, 2014b). L'appropriation par la grande distribution des valeurs associées à ces systèmes, processus émergents, réinterroge à nouveau la capacité des éleveurs à innover sur le plan technique, organisationnel et institutionnel pour rester maîtres de ces stratégies de segmentation.

\section{Conclusion}

Dans un marché libéralisé, les filières viande ovine et bovine, lait de vache, de brebis et de chèvre se transforment, entrainant une modification des rapports de force entre les opérateurs des différents maillons. Le pilotage des filières s'est, depuis plusieurs années, déplacé vers l'aval. Le partage de la valeur et la rémunération des producteurs sont en question. Les stratégies de segmentation de l'offre, parfois hybridées entre elles, et depuis peu, à l'initiative, pour certaines, des consommateurs, de plus en plus sensibles à ces questions, pourraient constituer, dans certaines conditions, des leviers pour les producteurs dans la négociation avec les opérateurs de l'aval. Elles peuvent aller de pair avec la structuration d'organisations collectives qui les renforcent.

L'ensemble de ces mutations incitent à la transformation des activités d'élevage. En effet, la performance économique des systèmes d'élevage résulte certes des choix techniques des éleveurs, mais aussi de leur capacité à faire face à la concurrence horizontale, à la pression concurrentielle exercée par les clients et les fournisseurs, à celle venant des produits substituts, ainsi qu'à gérer les modifications de l'environnement (Porter, 1999). La viabilité des élevages, et en particulier les modalités d'élaboration des revenus et de la gestion de la trésorerie, est donc liée aux conditions d'accès aux marchés, que nous venons d'analyser. Ceci induit pour les éleveurs la nécessité d'une certaine réappropriation de la commercialisation comme fonction de leurs systèmes d'élevage. Réfléchir aux choix de type de produits, de circuits et finalement de couples produits-acheteurs, présente des avantages, des écueils, crée des opportunités et génère des contraintes spécifiques (Nozières, 2014). Au côté de stratégies d'éleveurs où une seule forme de valorisation est recherchée, d'autres existent, s'appuyant sur une diversité de couples produit-acheteur issue d'un même élevage. Ces choix en matière de commercialisation nécessitent des évolutions dans le métier d'éleveur et en particulier lorsque celui-ci fait le choix principalement du CC puisqu'il doit posséder d'autres compétences commerciales voire managériales, mais aussi techniques. Enfin, les choix de produits et de circuits peuvent nécessiter l'adoption d'un cahier des charges qui, même s'il ne transforme pas les pratiques lors de leur conception (voir l'exemple des labels rouges en viande bovine, Roche et al., 2000), les fixent. En réalité, les liens entre choix de commercialisation et organisation des élevages sont assez complexes générant souplesses et rigidités (Nozières, 2014). Néanmoins, comme le mouvement de fond de l'élevage français (agrandissement des structures, spécialisation et concentration territoriale, intensification) demeure, ceci interroge la coexistence des systèmes productifs.

Toutefois, plusieurs questions demeurent : les alternatives aux filières dominantes peuvent elle connaître un réel essor (et à quelles conditions ?) ou resteront-elles des niches ? La montée en puissance des enjeux environnementaux constitue-t-elle une opportunité ? Quelles sont les capacités des éleveurs à innover sur le plan technique et organisationnel pour intégrer de nouvelles démarches et/ou favoriser leur diffusion? Comment le développement de l'économie collaborative associée à une numérisation des relations « rapprochant » consommateurs et producteurs transforme les filières ? Enfin, pour être opérants et favoriser le passage « de l'assiette aux champs ", les outils de mutualisation et de création de ressources que nous avons analysés, nécessitent d'être soutenus et encadrés par des dispositifs institutionnels tenant compte de la spécificité et de la diversité des territoires et des filières. Ceci réinterroge les choix de politiques publiques aux échelles locales, nationales et européennes pour accompagner la coexistence et l'évolution d'une diversité de systèmes productifs faisant vivre une diversité de territoires, mais aussi la détermination des consommateurs et des citoyens à assumer ces voies de développement ainsi que, réciproquement, la capacité des éleveurs à s'organiser en ce sens.

\section{Références}

Agreste, 2012. Un producteur sur cinq vend en circuit court. Agreste Primeur. 275, $4 p$.
Agreste, 2015. GraphAgri ,2015. Ministère de l'agriculture et de l'alimentation.
Agreste, 2016. GraphAgri, 2016. Ministère de l'agriculture et de l'alimentation. 
Agreste, 2017. GraphAgri, 2017. Ministère de l'agriculture et de l'alimentation.

Allain M.L., Chambolle C., 2003. Les relations entre la grande distribution et ses fournisseurs : bilan et limites de trente ans de régulation. Rev. Française Éco., 17, 169-212.

Ares G., Deliza R., 2010. Studying the influence of package shape and colour on consumer expectations of milk desserts using word association and conjoint analysis. Food Qual. Pref., 21, 930-937.

Aubron C., Peglion M., Nozières M.O., Boutonnet J.P., 2014. Démarches qualité et pastoralisme en France. Synergies et paradoxes. Rev. Géo. Alpine, 102, 17p.

Barraud-Didier V., Henninger M.C., Anzalone G., 2012. La distanciation de la relation adhérent-coopérative en France. Études Ru., 2, 119-130.

Baritaux V., Houdart M., 2015. Relations fournisseurs - grande distribution dans les filières agro-alimentaires. Une analyse de la trajectoire d'une démarche " filière de qualité ». Écon. Rurale, 346, 15-30

Baritaux V., Billion C., 2016. Les intermédiaires de la distribution dans la relocalisation des systèmes alimentaires : perspectives de recherche. In: $11^{\mathrm{e}}$ Congrès RIODD, Saint-Étienne, France, 6-8 juillet 2016.

Barjolle D., Sylvander B., 2003. Facteurs de succès des produits d'origine certifiée dans les filières agro-alimentaires en Europe : marché, ressources et institutions. INRA Prod. Anim., 16, 289-294.

Benkahla A., Boutonnet J.P., Fort F., 2005. Enjeux de la certification d'origine et des stratégies d'acteurs : le cas de I'AOC. Éco. SoC., 5, 877-894.

Bergès-Sennou F., Caprice S., 2003. Les rapports producteurs-distributeurs : fondements et implications de la puissance d'achat. Éco. Rur., 277, 192-205.

Berger Y., Marchal Y., Champanhet F., Riou Y., 2015. Mise en œuvre de la contractualisation dans la filière laitière française. Rapport CGAAER, 80p annexes.

Beylier R.P., Messeghem K., Fort F., 2012. Rôle des MDD de terroir dans la construction de la légitimité des distributeurs. Decisions Marketing, 66, 35-45.

Billaudeau V., Thareau B., 2010. «L'éleveur et I'oiseau »: rayonnement d'une démarche agroenvironnementale innovante. Marché et organisations, 1, 155-187.

Birlouez E., 2012. La viande dans les cultures alimentaires: du désir au tabou. Médecine et Nutrition, 48, $38-42$.

Boichard J., 1972. Le lait et les problèmes de l'élevage laitier en France. Rev. Géo. Lyon, 47, 99-135.

Bouamra-Mechemache Z., Duvaleix-Tréguer S., Ridier A., 2015. Contrats et modes de coordination en agriculture. Éco. Rur., 1, 7-28.

Boutonnet J.P., Napoléone M., Rio M., Monod F., 2005. AOC Pélardon, filière en émergence. Enseignements et questions vives. In : Symposium du programme PSDR
Pour et Sur le développement régional, Lyon, France, 9-11 mars 2005. 13p.

Boyer P., Cadilhon J.P., Depeyrot J.N., Ennifar M. Soler L.G., 2013. Le suivi des prix et des marges pour l'analyse de la formation des prix au détail des produits alimentaires : les cas du lait et de la viande bovine. Notes et études socio-économiques du MAAF, 32p.

Bowen S., 2010. Embedding Local Places in Global Spaces: Geographical Indications as a Territorial Development Strategy. Rural Socio., 75, 209-243.

Burch D., Dixon J., Lawrence G., 2013. Introduction to symposium on the changing role of supermarkets in global supply chains: from seedling to supermarket: agri-food supply chains in transition. Agric. Human Values, 30, 215-224.

Brulotte R.L., Di Giovine M.A., 2016. Edible identities: Food as cultural heritage. Routledge.

Cadilhon J., Dedieu M.S., 2011. Les organisations interprofessionnelles : un outil répandu de gestion des filières. Analyse du Centre d'Études et de Prospective, 31

Capt D., Lepicier D., Leseigneur A., 2014. Le rôle des territoires de projets infra-régionaux sur l'agriculture et l'alimentation. Le cas du développement de circuits de proximité. Géocarrefour, 89, 105-113.

Cechin A., Bijman J., Pascucci S., Omta 0., 2013. Decomposing the member relationship in agricultural cooperatives: Implications for commitment. Agribusiness, 29, 39-61.

Chabrat S., 2015. Collective marketing initiatives: an association of material, identity and organizational conditions of territory. The case of "Fin Gras du Mézenc". Semestrale di Studi e Ricerche di Geografia

Chatellier V., Jacquerie V., 2004. La diversité des exploitations laitières européennes et les effets différenciés de la réforme de la PAC de juin 2003. INRA Prod. Anim., 17, 315-333.

Chameroy F., 2013. Les effets du label sur la qualité perçue, les relations à la marque et le consentement à payer. Thèse de doctorat. Université d'Aix Marseille. $478 \mathrm{p}$.

Chazoule C., Fleury P., Brives H., 2015. Systèmes alimentaires du milieu et création de chaines de valeurs : concepts et études de cas dans la région Rhônes-Alpes. Éco. Soc., 37, 1203-1219.

Chiffoleau Y., Gauche A., Ollivier D., 2013. Impacts sociaux des circuits courts alimentaires sur les exploitations agricoles. Diversité des modèles et analyses croisées. Rapport Casdar RCC. 24p.

Chiron J., 2015. Biolait: des paysans producteurs et vendeurs de lait bio, pour un commerce équitable au Nord aussi. Pour, 3, 127-140.

Cinquegrana P., 2008. The Need of Transparency in Commodity and Commodity Derivatives Markets. ECMI Research Report.
Combris P., Maire B., Réquillart V., Caillavet F., Champenois A., Dury S., Gojard S., 2011. Chapitre 2 Consommation et consommateurs. Pour une alimentation durable. Réflexion stratégique duALIne. Esnouf C., Russel M., Bricas N. (Eds). Rapport InraCirad France, 26-43.

COM866, 2013. Report from the commission to the European Parliament and the Council on the case for local farming and direct sales labelling scheme. 12p.

Coquart D., Soufflet J.F., 1989. Dynamique de la concurrence et évolution de la filière viande bovine. Éco. Rur., 194, 15-22

Daumas J.C., 2006. Consommation de masse et grande distribution: une révolution permanente (19572005). Vingtième siècle, Revue d'histoire, 91, 57-76.

Delanoue E., Roguet C., 2015. Acceptabilité sociale de l'élevage en France : recensement et analyse des principales controverses à partir des regards croisés de différents acteurs. INRA Prod. Anim, 28, 39-50.

De Fontguyon G., Giraud-Héraud E., Rouached L., Soler L.G., 2003. Qualité des produits alimentaires et marques de filières. Socio. du travail, 45, 77-94.

De Fontguyon G., Sans P., 2003. L'industrie de transformation de la viande bovine en France : une approche historique (1950-2003). Cahier Inra Loria, 2003-07

de Kermadec C., 2011. Le commerce alimentaire spécialisé : déclin enrayé ? INSEE Première, 1359.

Dervillé M., Vandenbroucke P., Bazin G., 2012. Suppression des quotas et nouvelles formes de régulation de l'économie laitière: les conditions patrimoniales du maintien de la production laitière en montagne. Revue de la régulation, Capitalisme, institutions, pouvoirs, 12

Dervillé M., Allaire G., 2014a. Quelles perspectives pour les filières laitières de montagne après la suppression des quotas laitiers ? une approche en termes de régime de concurrence. In :Spécificités de l'élevage de ruminants en montagne. Grosclaude J., Thibier M., Baumont R. (Eds). Dossier, INRA Prod. Anim., 27, 17-30.

Dervillé M., Allaire G., 2014b. Change of competition regime and regional innovative capacities: Evidence from dairy restructuring in France. Food Policy, 49, 347-360.

Dervillé M., Wallet F., 2014. Institutionalizing short food supply chains for sustainable development: challenging issues. Intern. Agric. Pol., 2, 21-32.

Dervillé M., Lambaré P., You, G., 2016. Accès au marché des producteurs après la suppression des quotas laitiers : une analyse de la diversité des contrats et des organisations de producteurs. Renc. Rech. Rum., 23, 195-198.

Etchevarria L., 2013. Produire et commercialiser de la viande bovine en circuits courts. Casdar RCC. 28p.

Filippi M., Triboulet P., 2006. Coordination des acteurs et valorisation de produits liés à l'origine. Les signes d'identification comme signes d'exclusion? Rev. Éco. Rég. Urb., 1, 103-129. 
Filippi M., Frey 0., Mauguet R., 2008. Les coopératives agricoles face à l'internationalisation et à la mondialisation des marchés. Rev. Intern. Éco. Soc., 310, 31-51.

Filippi M., Triboulet P., 2011. Alliances stratégiques et formes de contrôle dans les coopératives agricoles. Revue d'économie industrielle, 133, 57-78.

Font i Furnols M., Realini C., Montossi F., Sanudo C., Campo M.M., Oliver M.A., Nute G. R., Guerrero L., 2011. Consumers purchasing intention for lamb meat affected by country of origin, feeding system and meat price: A conjoint study in Spain, France and United Kingdom. Food Qual. Pref., 22, 443-451.

Fumey G., Bérion P., 2010. Dynamiques contemporaines $d^{\prime}$ un terroir et $d^{\prime}$ un territoire : le cas du gruyère de Comté. Ann. Géo, 4, 384-403.

France AgriMer, 2010. La consommation française de viandes. Évolutions depuis 40 ans et dernières tendances. Les synthèses de France AgriMer, 1. 8p.

France AgriMer, 2012. La filière bovine française face à la sortie des quotas laitiers. Les synthèses de France AgriMer, 12, 12p.

France AgriMer, 2015a. Les produits carnés, avicoles et laitiers. Données statistiques 2014. France / Union européenne/Monde. Données et bilans. 200p.

France AgriMer, 2015b. Impact de la crise économique sur la consommation de viandes et évolutions des comportements alimentaires. Les synthèses de France AgriMer, 21, 15p.

France AgriMer, 2016a. Les filières animales terrestres et aquatiques : bilan 2015, perspectives 2016, 137p.

France AgriMer, 2016b. La transformation laitière française. État des lieux et restructuration Données 2014, évolution 2002/2008/2014, 47p.

Galliano D., 1995. Les groupes industriels de l'agroalimentaire français, INRA-Economica.

Grunert K.G., Valli C., 2001. Designer-made meat and dairy products: consumer-led product development. Livest. Prod. Sci., 72, 83-98.

Guiomar X., 2011. Les collectivités locales à la recherche d'une agriculture de proximité. Pour, 2 , 169-183.

Hansen M.H., Morrow Jr J.L., Batista J.C., 2002. The impact of trust on cooperative membership retention, performance, and satisfaction: an exploratory study. Intern. Food Agribus. Manag. Rev., 5, 41-59.

Harper G., Henson S., 2001. Consumer Concerns about Animal Welfare and the Impact on Food Choice. Final report. EU FAIR CT98-3678. 38p.

Henning D.R., Baer R.J., Hassan A.N., Dave R., 2006. Major Advances in Concentrated and Dry Milk Products, Cheese, and Milk Fat-Based Spreads. J. Dairy Sci., 89, 1179-1188.

Idele, 2016a. L'année économique ovine. Perspectives 2016. Des Signaux contradictoires. Dossier Économie de l'élevage, 467, 40p.
Idele, 2016b. 2015 : I'année économique viande bovine. Perspectives 2016. Dossier Économie de lélevage, $464,44 p$.

INSEE, 2017. La situation du commerce en 2016 Rapport établi pour la Commission des comptes commerciaux de la Nation ", Documents de travail $\mathrm{n}^{\circ} \mathrm{E} 2017 / 04$.

Jeanneaux P., Meyer D., Barjolle D., 2011. Gouvernance des filières fromagères sous $\mathrm{AOP}$ et origine des prix de lait : un cadre d'analyse. Journ. Rech. Sci. Soc., Dijon, France, 8 .

Laisney C., 2011. L'évolution de I'alimentation en France. Tendances émergentes et ruptures possibles. Futuribles, 372, 5-23.

Laisney C., 2013. Les différences sociales en matière d'alimentation. Analyse. CEP. 64. 4p.

Legendre V., Sans P., Barrey S., Boutin B., 2017. Controverses sur la consommation de viande : enseignement d'une analyse sociologique. Inra Prod. Anim., 30, 479-486.

Lepers X., 2003. La relation d'échange fournisseurs-grand distributeur : vers une nouvelle conceptualisation. Rev. Française Gestion, 2, 81-94.

Mainsant P., 2012. Dans l'industrie de la viande depuis 1986, série noire pour les grands groupes coopératifs. Demeter, 305-314.

Marty S., Petit N., Reffay M., 2015. La contractualisation dans le secteur bovin. Rapport CGAAER n ${ }^{\circ} 14099$, $73 p$.

Ménard C., 2003. Économie néo-institutionnelle et politique de la concurrence les cas des formes organisationnelles hybrides. Éco. Rur., 277, 45-60.

Mevel 0., 2010. Les relations entre la production et la distribution : le cas du partage de la valeur ajoutée dans la filière laitière française. Gérer et comprendre, 101, 38-49

Michaëlsson K., Wolk A., Langenskiöld S., Basu S., Lemming E.W., Melhus H., Byberg L., 2014. Milk intake and risk of mortality and fractures in women and men: cohort study. Br. Med. J., 349.

Michaud C., 2015. Les ateliers de transformation collectifs comme éléments de construction de systèmes alimentaires durables? In : Ateliers de transformations collectifs. Mundler P., Valorge F. (Edts). Educagri, 259-274

Nichèle V., Andrieu E., Boizot C., Caillavet F., Darmon N., 2005. La consommation d'aliments et de nutriments en France. Évolution 1969-2001 et déterminants socio-économiques des comportements. Document de travail 05-07, Inra SES, 133p.

Norat T., Riboli E., 2003. Dairy products and colorectal cancer. A review of possible mechanisms and epidemiological evidence. Eur. J. Clin. Nut., 57, 1-17.

Noury J.M., De Fontguyon, G., Sans P., 2005. La construction collective de la qualité sur un territoire : l'exemple de l'appellation « Maine-Anjou » en viande bovine. INRA Prod. Anim., 182, 111-118.
Napoléone M., Corniaux C., Leclerc B., 2015. Voies lactées : dynamique des bassins laitiers entre globalisation et territorialisation. Cardère, 320p.

Nozières M.0., 2014. La commercialisation des produits, source de flexibilité pour les éleveurs? le cas de l'élevage ovin allaitant en Languedoc-Roussillon. Thèse de doctorat de Montpellier SupAgro, France, 200p.

Nozières M.O., Boutonnet J.P., Petit T., Galan E., 2015. Commercialisation de la viande ovine dans l'arrière-pays méditerranéen français. In: The value chain of Mediterranean sheep and goat products. Options méditerranéennes, Series A, 115, 119-123.

Perrot C., Béguin E., Morhain B., Tchakérian E., 2005. Lélevage dans les exploitations françaises. État des lieux et perspectives. Éco. Rur., 288.

Perrot C., Chatellier V., Gouin D.M., Richard M., You G., 2016. Le secteur laitier français est-il compétitif face à la concurrence européenne et mondiale? In : Colloque SFER. Clermont-Ferrand, France, 9-10 juin 2016.

Pion I., 2016. État des lieux de l'accès au marché certification sanitaire. In : Conférences Les marchés mondiaux du lait et de la viande en 2016. Paris, France.

Porter M.E., 1999. La concurrence selon Porter. Village mondial.

Rastoin J.L., 2000. Une brève histoire de l'industrie alimentaire. Éco. Rur., 255-256, 61-71.

Rastoin J.L., 2008. Les multinationales dans le système alimentaire. Rev. Proj., 6, 307, 61-69.

Ricard D., 2014. Les mutations des systèmes productifs en France : le cas des filières laitières bovines. Rev. Géo. Est, 54.

Rigolot C., 2016. Le capital social des filières : une dimension essentielle de leurs capacités d'adaptation. Illustration avec la filière comté. Cah. Agric., 25, 45007 .

Roberts S.D., Micken K.S., 1996. « Le Fromage As Life: French Attitudes and Behavior Toward Cheese ", in NA - Advances in Consumer Research, 23. Eds. Kim P. Corfman and John G. Lynch Jr., Provo, UT: Assoc. Consumer Res., 111-119.

Roche B., Dedieu B., Ingrand S., 2000. Analyse comparative des cahiers des charges Label Rouge gros bovins de boucherie. Renc. Rech. Rum., 7, 259-262.

Roosen J., Lusk J.L., Fox J.A., 2003. Consumer demand for and attitudes toward alternative beef labeling strategies in France, Germany, and the UK. Agribusiness, 19, 77-90.

Sans P., Coquart D., 1998. Grande distribution alimentaire et changements organisationnels. Le cas de l'industrie de transformation de la viande bovine. Éco. Rur., 245-246, 111-118.

Sans P., De Fontguyon G., 1999. Différenciation des produits et segmentation de marché : l'exemple de la viande bovine en France. Cah. Éco. Socio. Rur., 5 , 55-76. 
Sans P., 2003. La qualité différenciée de la viande bovine. La nécessaire stratégie d'innovation. Biothechnol. Agron. Soc. Environ., 7, 151-160.

Setbon M., Raude J., Fischler C., Flahault A., 2005. Risk perception of the "mad cow disease" in France: determinants and consequences. Risk Analysis, 25, 813-826.

Soufflet J.F., 1990. Compétitivité et stratégies agro-industrielles dans la filière viande bovine européenne en constitution. Éco. Rur., 197, 42-48.

Sylvander B., 1996. Normalisation et concurrence internationale : la politique de la qualité alimentaire en Europe. Éco. Rur., 231, 56-61.
Tavoularis G., Recours F., Hebel P., 2007. Perception de la qualité et des signes officiels de qualité dans le secteur alimentaire. Credoc. Cah. Rech., 236.

Torre A., Galliano D., Rama R., 2013. Localisation et ancrage territorial des firmes agroalimentaires Introduction au dossier thématique, Économies et Sociétés, Série « Systèmes Agroalimentaires », 11-12.

Trouvé A., Dervillé M., Gouin D.M., Pouch T., FinkKessler A., Kroll J.C., Rat-Aspert 0., Briot X., Lambaré $P ., 2016$. Synthèse : Étude sur les mesures contre les déséquilibres de marché: Quelles perspectives pour l'après quotas dans le secteur laitier européen?, Marché référencé SSP-DGPAAT-2014-027, 25p.
Veerman C., Giesen H., Paumier A.L., Dobbin D., Babuchowski A., Šarmír I., Hogan P., Valverde E., Juhasz A., Iwarson T., Fresco L.0., Bédier B., Calzolari $G ., 2016$. Improving market outcomes: enhancing the position of farmers in the supply chain. European Task Force. 76p.

Verbeke W., Van Wezemael L., de Barcellos M.D, Kügler J.0., Hocquette J.F., Ueland 0., Grunert K., 2010. European beef consumers' interest in a beef eating-quality guarantee: Insights from a qualitative study in four EU countries. Appetite, 54, 289-296

You G., 2015. Contractualisation et modes de coordination dans la filière laitière. Éco. Rur., 1, 87-100.

\section{Résumé}

Cette synthèse propose une analyse des grandes transformations des filières de produits carnés et laitiers pour comprendre quelle y est la place des éleveurs. Elle s'appuie sur une recherche bibliographique ainsi que sur des cas d'études concernant les transformations de ces filières. Dans un premier temps, nous dégageons les principaux mouvements en matière de demande des produits laitiers et carnés. Dans un second temps, nous examinons les recompositions très fortes au sein des filières sous l'effet de la libéralisation des marchés : concentration, contractualisation. Puis, le troisième temps analyse la place des éleveurs dans ces transformations, en particulier dans la structuration des filières et la segmentation de l'offre à destination des consommateurs.

\section{Abstract}

\section{Transformations of the French meat and dairy product sectors: the role of breeders}

This synthesis provides an analysis of the major transformations of the meat and dairy product sectors to understand the role of breeders. It refers to a literature review and to some case studies on transformations in the bovine and ovine meat sectors and bovine, ovine and caprine milk sectors. First, we present the main movements regarding the demand of dairy and meat-based products. Secondly, we examine the very strong reorganizations of these value chains under the influence of the markets' liberalization: concentration, contractualization. Then, the third step analyzes the place of the breeders in these transformations, in particular in the structuring of the value chains and what is their role in the segmentation of the offer to consumers.

NOZIÈRES-PETIT M.-O., BARITAUX V., COUZY C., DERVILLÉ M., PERROT C., SANS P., YOU G.; 2018. Transformations des filières françaises de produits carnés et laitiers : la place des éleveurs en question. INRA Prod. Anim., 31, 69-82. 